\section{Minimum database, one flashback is all it takes}

\section{Holly Witchell}

Minimum database consists of a few diagnostic tests which help aid in a rapid assessment of a patient admitted into the hospital or practice, used alongside a clinical examination.

Packed cell volume (PCV) and total solids (TS) can tell us a lot from a very small amount of blood. PCV tells us the percentage of red blood cells (RBC) in circulation and TS tells us a measured amount of plasma proteins within circulation. These can add to our picture of the patient's overall clinical status, that is, dehydration, anaemia via haemorrhage or haemolysis, hypoproteinaemia or icterus.

Blood smears can quickly show us if patients are regenerating $\mathrm{RBCs}$, signs of immune-mediated disease, haemoplasma, thrombocytopenia and whether we have a neutrophilia or neutropenia.

Blood glucose only needs a very small amount of blood and can be critical in indicating whather to supplement glucose or not in collapsed or critical patients.

Lactate can be measured by a handheld monitor similar to a glucometer. Lactate is created by anaerobic respiration of cells within the body; this can be high in patients with hypoperfusion or hypovolaemia and can help direct care and fluid therapy for patients. Lactate can be a useful indicator in helping diagnose septic peritonitis: abdominal fluid can be compared to the patient's serum, a septic patient's abdominal fluid will have a blood glucose $>1.1 \mathrm{mmol} / \mathrm{l}$ lower than that of serum and a lactate $>2 \mathrm{mmol} / \mathrm{l}$ higher than that of serum.

Measuring electrolytes can be lifesaving in critical patients, in cases such as urethral obstruction, Addisonian crisis, gastric foreign body or hypocalcaemia. These may give us clues as to what is happening within our patient or tell us something that needs correcting straight away.

Blood gases and acid-base balance only require a small amount of blood from our patient, some machines can also run electrolytes at the same time. Blood gases can tell us a lot about a patient's respiratory function with regard to carbon dioxide and oxygenation, this can be useful in clarifying a hypoxia due to a respiratory dysfunction or a ventilation issue causing hypo-/ hypercapnia. Acid-base balance tells us about the patient's metabolic status and can guide us through fluid therapy needed and help diagnose certain metabolic issues such as diabetic ketoacidosis

Blood urea nitrogen (BUN) can be ran as a single test in-house. A raised BUN can point towards gastrointestinal bleed due hypovolaemia causing a pre-renal azotaemia or renal/ liver dysfunction, as urea is produced in the liver and excreted via the kidneys.

\section{KEY LEARNING OBJECTIVES}

- Learn what tests are performed in the minimum database

- Understand use of minimum database results and apply this to patients in practice

- Be aware of what we can gain from minimum database in our emergency patients

\section{MULTIPLE CHOICE QUESTIONS}

1. What is the most likely cause for a sudden decrease in TS and a normal PCV indicate?
(A) Dehydration
(B) Haemorrhage
(C) Anaemia
(D) Hypoproteinaemia

2. Which type of abnormal RBC would you commonly see on a blood smear for immune-mediated haemolytic anaemia?
(A) Heinz bodies
(B) Acanthocytes
(C) Spherocytes
(D) Schistocytes

3. In a urethral obstruction, which is most common electrolyte abnormality?
(A) Sodium
(B) Calcium
(C) Phosphorus
(D) Potassium

\section{Major body system assessments}

\section{Jessica Herley}

The goal of performing an MBSA is to efficiently identify any potential threats to a patient's life. An MBSA is a rapid clinical examination of a patient, during which the cardiovascular, respiratory and central nervous systems are evaluated. This approach is somewhat different from the head-to-tail physical examination commonly performed that is only appropriate for non-emergency patients Abnormalities detected whilst carrying out an MBSA should be dealt with immediately. Evaluation of the patient's major body systems forms the core for that individual's ongoing care and an MBSA is usually repeated at regular intervals to assess disease progression and to assess how the patient is responding to treatment.

\section{ASSESSMENT OF THE CARDIOVASCULAR SYSTEM}

The cardiovascular assessment should include cardiac auscultation. Cardiac auscultation is performed to assess heart rate and rhythm and to detect the presence of a 
cardiac murmur. Peripheral pulse quality should be assessed and any pulse deficits should be noted. Mucous membrane colour and capillary refill time should be assessed to evaluate the patient's perfusion status.

\section{ASSESSMENT OF THE RESPIRATORY SYSTEM}

Before handling the emergency patient, visual observations of the patient's chest wall will provide information including respiratory rate, and it can be determined whether the patient has an increased respiratory effort. If the patient is suffering from respiratory distress then oxygen therapy should be administered whilst the patient undergoes any further examination. Thoracic auscultation should be performed to assess for the presence of any abnormal lung sounds.

\section{ASSESSMENT OF THE NEUROLOGICAL SYSTEM}

The neurological assessment of an emergency patient involves assessing the patient's demeanour, whether the patient is able to ambulate and whether the patient has any cranial nerve deficits.

\section{KEY LEARNING OBJECTIVES}

- Understand when it is appropriate to perform a major body system assessment (MBSA)
Understand why we perform MBSAs

Understand how to perform an MBSA

\section{MULTIPLE CHOICE QUESTIONS}

1. During an MBSA of a feline patient, you note that the patient's heart rate is $100 \mathrm{bpm}$. Bradycardia (heart rate less than $120 \mathrm{bpm}$ ) in a cat is most likely to be associated with which of the following conditions?

(A) Allergic bronchitis (feline asthma)

(B) Aortic thromboembolism

(C) Hypovolaemic shock

(D) Severe pain

2. What lung sounds may be noted during thoracic auscultation for a patient that has a pneumothorax?

(A) Increased lung sounds

(B) Increased heart sounds

(C) Decreased or absent lung sounds

(D) Crackles

3. A patient is presented with a suspected gastric dilatation-volvulus. Which of the following is least likely to be a problem?
(A) Hypotension
(B) Cardiac arrhythmias
(C) Cerebral oedema
(D) Respiratory acidosis

\section{Pleural space disease: what are you doing there?}

\section{Jessica Herley}

The pleural space is defined as the area between the lungs and the chest wall. Pleural space disease occurs when air, fluid or tissue occupies this space. Examples of pleural space disease include pneumothorax (air in the pleural space) and pleural effusion (fluid in the pleural space). Pleural space disease may be unilateral or bilateral, depending upon the cause.

Clinical signs of pleural space disease include increased respiratory rate and effort, coughing, fever, weight loss and lethargy. Clinical examination of patients in respiratory distress should include thoracic auscultation during which dull lung sounds are most commonly noted if the patient is suffering from pleural space disease. A pleural effusion can occur secondary to heart disease so cardiac auscultation should also be performed to assess for evidence of a murmur or cardiac arrhythmia.
Pleural space disease can be diagnosed by taking radiographs of the thorax. Alternatively, or additionally, a TFAST (thoracic focused assessment with sonography for trauma) scan may be undertaken to check for the presence of air, tissue or fluid within the pleural space. Thoracocentesis is an invasive procedure that is performed to remove fluid or air from the pleural space. Thoracocentesis should be performed for any patient that has a build up of air or fluid in the pleural space which is affecting their respiratory function. Thoracentesis is a procedure which involves inserting a needle or catheter into the pleural space and withdrawing the air or fluid which has built up within the pleural space. The procedure usually provides immediate relief for the patient and if there is fluid present within the pleural space, this can be collected and sent for laboratory analysis. Patients in respiratory distress may object to the restraint that is required for thoracocentesis. Any distress suffered by a dyspnoeic animal can be life threatening so sedation or anaesthesia may be required to perform thoracocentesis safely. Coagulopathies are a contraindication of thoracocentesis, but sometimes the procedure cannot be avoided, especially in the case of life-threatening pleural haemorrhage.

Treatment of pleural space disease often includes oxygen therapy and the patient will require strict rest. 